\title{
The edges of human performance in psychiatry
}

Rajvinder Samra

\author{
Summary \\ Developing a realistic multifactorial model of human perform- \\ ance in psychiatry will better inform interventions targeting \\ clinician overwork and burnout, which contribute to risk and \\ error in medicine. This heralds a new approach, allowing better \\ detection by individuals, colleagues and automated systems, to \\ responding to degraded performance in psychiatry.
}

\section{Keywords}

Education and training; information technologies; clinical governance; social functioning; risk assessment.

\section{Copyright and usage}

(c) The Author 2020.
Rajvinder Samra is a Chartered Psychologist and a Lecturer in the School of Health, Wellbeing and Social Care at The Open University, UK

Psychiatry is grounded in complexity and uncertainty. Inherent challenges include characterising another individual's experiences and their meaning. The longitudinal course of these illness experiences is mapped to existing models of mental disorder and limited diagnostic systems. However, models of mental disorder differ across global and cultural communities, and across professional disciplines, ${ }^{1}$ underscoring the rise of person-oriented approaches to care. ${ }^{1}$ Crucially, these approaches deepen the challenges of work, inviting psychiatrists to grapple with human values and reconcile value conflicts between patients, professionals and multidisciplinary team members. ${ }^{1}$

Current approaches to understanding how psychiatrists manage their performance and the quality of their work in this dynamic environment are strikingly basic, focusing simply on events, such as adverse events or medical errors. We lack knowledge about the conditions and signs that clinicians are being hindered in their work; instead, we look simply for the outcomes that prevail after risky conditions have firmly taken root.

We currently know the conditions that affect the quality of complex work. Nine key factors underpin human performance in safety-critical workplace settings. ${ }^{2}$ These factors include stress, mental workload, attention, communication, vigilance, situational awareness, fatigue, teamwork and trust. Changes in one of these factors set off cascade reactions among the other factors to affect one's performance over time. ${ }^{2}$ For example, feeling highly fatigued reduces our vigilance and can lower our communication efforts with others, laying the foundations for mistakes and errors. To date, when considering work performance, we might note that an external stressor poses a risk to performance (for example, organisational cost-cutting), but we have failed to consider and model the downstream effects on the individual. How exactly does experiencing stress from organisational cost-cutting affect work quality, and how can this be identified as it is unfolding? For example, does stress typically or temporally affect attention, situational awareness, teamworking or communication? By modelling this, we can understand and identify the degradation of clinicians' work performance over time. ${ }^{3,4}$

Relationships between work factors are not always straightforward. Workload and stress both individually demonstrate curvilinear relationships with error, which means that work performance is better when they are moderately present. ${ }^{4}$ Low mental workload is associated with reduced vigilance (boredom). When mental workload is very high, we narrow our attentional focus to cope with the demand, placing ourselves at risk of missing important signs or events. ${ }^{4}$ Some work factor relationships are protective; staying alert and situationally aware mitigates against risk from high workload. ${ }^{4}$ Despite this knowledge, we have not researched how clinicians protect their performance in psychiatry to remain safe. This avenue of work could better inform junior clinician training so that it is more realistic to the role in actual practice.

Modelling human performance by using a multifactorial approach is a new paradigm in occupational safety research., ${ }^{2,4}$ Work factors typically cluster together in dyads or triads, creating error-prone conditions for decision-making. For example, having a very high workload creates the conditions for reduced teamwork and inadequate communication between individuals and teams. The risk to safety is compounded over time., ${ }^{2,4}$ The interactional nature of psychiatry, with both patients and colleagues, means that clinicians' situational awareness, communication, teamwork and trust are critical. These factors are necessary to gauge others' experiences and values, ${ }^{1}$ to provide high-quality person-centred care. Worsening clinician performance in these key factors is likely to have negative implications for patients and colleagues. There will be ramifications for whole teams as one clinician's performance degrades. Alternatively, if used constructively, degraded performance in these factors can serve as a warning indicator to others, offering the opportunity for other team members to address or mitigate risk.

Frankly, it is deeply concerning that there are currently no systematic ways of tracking degraded performance to identify clinicians working in high-risk conditions. To address this gap, we first need an understanding of how the nine work factors are linked and interact to affect work performance specifically in psychiatry. Second, we need to explore the typical trajectories from optimal to degraded performance, ${ }^{2}$ so we can better determine when a clinician is suffering burnout or at higher risk of making an error.

Identifying the edges of safe performance will involve asking clinicians to describe what happens psychologically, emotionally, physically and behaviourally when their performance is declining. How can they and others identify very risky performance? Physical, cognitive and behavioural markers, along with acute events, can collectively signpost the edge of safe performance. Researchers also need to examine whether clinicians losing situational awareness presents a unique concern in psychiatry. Specifically, whether a clinician's ability to perceive others' experiences and values (patients and colleagues) changes as their situational awareness degrades, and the effect of these changes on their clinical reasoning and treatment recommendations. 
Modelling what work looks like at the edge of performance can aid self-detection for clinicians. A range of changes will be identifiable to the clinician: physiological changes (faster heartbeat), cognitive changes (reduced self-awareness, easily distracted), subjective changes (feelings of losing control), verbal changes (negative words) and non-verbal changes (slouched position). ${ }^{3}$ Behavioural changes from declining performance tend to include performing reactively (falling behind and rushing tasks), narrowed attention (not attending to pertinent information), rapid or premature closure of decision-making and providing incorrect instructions or guidance. $^{3}$

We can, and should, do better in utilising the available information systems for detection of clinician overwork, burnout or highrisk conditions. We know that work in psychiatry requires high levels of accurate communication about medication and treatment, as well as safety risks ${ }^{5}$ to patients and staff. It stands to reason that degraded communication or teamworking performance from one team member under immense workload will (negatively) shape team performance and safety. Information systems underpinning team communication processes and patient data records could usefully support the identification of how error-prone teamworking environments manifest over time.

Common medication errors in psychiatry include omissions in computerised physician order entry relating to dosing regimens, dose, drug and treatment time. ${ }^{5}$ Highly performing teams rectify issues in time, such as nurses intercepting omission of pro re nata dosing regimens, ${ }^{5}$ but these are informal and unsystematic mitigation strategies by team members. Past attempts to reduce error still typically rely on increasing cognitive workload; for example, teaching residents a mnemonic for better hand-offs and incorporating it into the electronic health record. This approach actually increases the workload, and also fails to provide the individual with any performance intelligence that can empower them to identify and respond to increased risk. Instead, imagine a team member who learns that there are suddenly large deviations in the amount of information omissions or edits/interceptions in the patient records from those within the team and becomes alerted to a colleague working in error-prone conditions. Conversely and counterintuitively, lower-than-average edits and corrections may actually indicate a failure to correct information in time and may indicate the entire team is operating in error-prone conditions.

It is important to recognise that displays of psychological and behavioural change in clinicians working at the edge of safe performance may negatively affect the therapeutic relationship and care quality for patients receiving psychotherapy. Institutionalising recovery into existing workplaces will require training clinicians that there are edges to safe performance and, consequently, times where they must reduce their task loads and prioritise recovery or face greater risks of error, burnout or undermining their relationships with patients and colleagues.

The multifactorial modelling of clinicians' performance in psychiatry will be a new paradigm for conceptualising how this work is realistically done. In medicine more generally, the growing concerns about physician burnout as a hard outcome for human performance has also led to a polarisation of intervention stages: prevention and recovery from burnout - before and after. We have fundamentally overlooked how identifying and mapping human performance in psychiatry, as it degrades over time from multifactorial stressors, can uniquely address the forgotten process of what happens during burnout and how individuals and systems can identify and respond as it is happening.

Rajvinder Samra (D), PhD, School of Health, Wellbeing and Social Care, The Open University, UK

Correspondence: Rajvinder Samra. Email: Rajvinder.samra@open.ac.uk

First received 5 Sep 2019, final revision 7 Feb 2020, accepted 27 Feb 2020

Declaration of interest
None.
ICMJE forms are in the supplementary material, available online at https://doi.org/10.1192/bjp.
2020.59.
References

1 Fulford KW, Stanghellini G, Broome M. What can philosophy do for psychiatry? World Psychiatry 2004; 3: 130-5.

2 Silvagni S, Napoletano L, Graziani I, Le Blaye P, Rognin L. Concept for Human Performance Envelope. Future Sky Safety, 2015 (https://www.futureskysafety.eu/wp-content/uploads/2015/12/FSS_P6_DBL_D6.1-Concept-forHuman-Performance-Envelope_v2.0.pdf).

3 Edwards T, Sharples S, Kirwan B, Wilson J. Identifying markers of performance decline in air traffic controllers. In Human Factors in Transportation: Social and Technological Evolution Across Maritime, Road, Rail, and Aviation Domains (eds G Di Bucchianico, A Vallicelli, NA Stanton, SJ Landry): 367-84. CRC Press, 2016.

4 Edwards T, Homola J, Mercer J, Claudatos L. Multifactor interactions and the air traffic controller: the interaction of situation awareness and workload in association with automation. Cogn Technol Work 2017; 19: 687-98.

5 Soerensen AL, Lisby M, Nielsen LP, Poulsen BK, Mainz J. The medication process in a psychiatric hospital: are errors a potential threat to patient safety? Risk Manage Healthc Policy 2013; 6: 23-31. 